\title{
POLYNOMIAL RINGS OVER GOLDIE-KERR COMMUTATIVE RINGS
}

\author{
CARL FAITH \\ (Communicated by Eric Friedlander) \\ Dedicated to the memory of Pere Menal (1951-1991)
}

\begin{abstract}
All rings in this paper are commutative, and acc $\perp$ (resp., $\operatorname{acc} \oplus$ ) denotes the acc on annihilators (resp., on direct sums of ideals). Any subring of an acc $\perp$ ring, e.g., of a Noetherian ring, is an acc $\perp$ ring. Together, acc $\perp$ and $\operatorname{acc} \oplus$ constitute the requirement for a ring to be a Goldie ring. Moreover, a ring $R$ is Goldie iff its classical quotient ring $Q$ is Goldie.

A ring $R$ is a Kerr ring (the appellation is for J. Kerr, who in 1990 constructed the first Goldie rings not Kerr) iff the polynomial ring $R[x]$ has acc $\perp$ (in which case $R$ must have acc $\perp$ ). By the Hilbert Basis theorem, if $S$ is a Noetherian ring, then so is $S[x]$; hence, any subring $R$ of a Noetherian ring is Kerr.

In this note, using results of Levitzki, Herstein, Small, and the author, we show that any Goldie ring $R$ such that $Q=Q_{c}(R)$ has nil Jacobson radical (equivalently, the nil radical of $R$ is an intersection of associated prime ideals) is Kerr in a very strong sense: $Q$ is Artinian and, hence, Noetherian (Theorems 1.1 and 2.2). As a corollary we prove that any Goldie ring $A$ that is algebraic over a field $k$ is Artinian, and, hence, any order $R$ in $A$ is a Kerr ring (Theorem 2.5 and Corollary 2.6). The same is true of any algebra $A$ over a field $k$ of cardinality exceeding the dimension of $A$ (Corollary 2.7).

Other Kerr rings are: reduced acc $\perp$ rings and valuation rings with acc $\perp$ (see 3.3 and 3.4).
\end{abstract}

\section{INTRODUCTION}

A property of a Goldie ring $R$ needed for the proof is found in [F2, Corollary 3.7], which states that any ring $R$ with the acc on annihilator ideals (= $\operatorname{acc} \perp$ ) has semilocal quotient ring $Q$. Another property needed is the theorem of Levitzki [Le] and Herstein and Small [HS]: any nil ideal in a (two-sided) acc $\perp$ ring is nilpotent. Finally we need a result of the author in [F1]: Any finitely embedded acc $\perp$ ring is Artinian.

Received by the editors March 30, 1992.

1991 Mathematics Subject Classification. Primary 13B25, 13E05, 13E10; Secondary 16P60.

Pere Menal died April 4, 1991. A Memorial Service was held at Universitat Autónoma de Barcelona, and a memorial booklet contains the reminiscences of two of his students, Jaume Moncasi and Pere Ara (who were also on the Faculty with Pere), and of his Ph.D. adviser, Manuel Castellet, Director of the Centre Recerca de Matemática at U.A.B. Also see the Pere Menal Memorial Volumes [P]. 
Any subring of an acc $\perp$ ring satisfies acc $\perp$; however, the same does not hold for the second defining condition of Goldie rings: $(\operatorname{acc} \oplus)=$ acc on direct sums of ideals of $R$, a subring of a right Noetherian ring need not have $\operatorname{acc} \oplus$.

\section{Proof of Theorem 1.1}

We first prove Theorem 1.1 and use it to prove Theorem 2.2.

1.1. Theorem. A commutative ring $R$ is Artinian iff $R$ is a Goldie quotient ring with nil Jacobson radical. ${ }^{1}$

Proof. The necessity is classical. Conversely, as noted, any acc $\perp$ ring $R$ has a semilocal (Kasch) quotient $Q=Q_{c}(R)$. Moreover, in any acc $\perp$ ring, every nil ideal is nilpotent [HS, Le]. Thus, $R=Q$ is semiprimary and, hence, has essential socle $S$. By acc $\oplus,|S|<\infty$; hence, $R$ is a finitely embedded acc $\perp$ ring. By [F1], $R$ is Artinian

1.2. Corollary. If $R$ is a commutative Goldie ring, with zero-dimensional $Q=$ $Q_{c}(R)$, then $Q$ is Artinian and, hence, $R$ is Kerr.

\section{THE ASSOCIATED PRIME RADICAL}

The intersection of all prime ideals is a nil ideal $N(R)$ called the prime or nil radical. We let Ass $R$ denote the set of associated prime ideals; Ass prime $\operatorname{rad} R$ denotes the intersection

$$
\text { Ass prime } \operatorname{rad} R=\bigcap_{P \in \text { Ass } R} P \text {. }
$$

We also let $\operatorname{Max} R$ denote the set of maximal ideals of $R$. Then $J(R)=$ $\bigcap_{M \in \operatorname{Max} R} M$ is the Jacobson radical. A ring $R$ is a Kasch ring provided that $\operatorname{Max} R \subseteq$ Ass $R$.

2.1. Proposition. If $R$ is a Kasch ring, then

$$
\text { Ass } R=\operatorname{Max} R \text {; }
$$

hence,

$$
J(R)=\text { Ass prime } \operatorname{rad} R .
$$

Moreover, $R=Q_{c}(R)$.

Proof. This follows from the triviality that every ideal $\neq R$ is contained in a maximal ideal, so if $P$ is an associated prime and $R$ is Kasch, then $P \in \operatorname{Max} R$. This proves (1) and (2). If $x$ is a unit of $R$, then $x R \neq R$; hence, $x R$ is contained in some $M \in \operatorname{Max} R$, so $x$ is a zero divisor. Thus $R=Q_{c}(R)$.

Note. As stated, any acc $\perp$ ring $R$ has (semilocal) Kasch $Q_{c}(R)$ [F2, Corollary 3.7]. This is used in the proof of the following:

2.2. Theorem. $A$ ring $R$ has Artinian $Q_{c}(R)$ iff $R$ is a Goldie ring with nil Ass prime $\operatorname{rad} R$, that is, with

$$
\text { Ass prime } \operatorname{rad} R=\operatorname{nil} \operatorname{rad} R \text {. }
$$

\footnotetext{
${ }^{1}$ Theorem 1.1 was proved in the Addendum [F1].
} 
Proof. If $I$ is an ideal of $R$, then $I$ is an annihilator of $R$ iff $I Q$ is an annihilator of $Q$. Hence, $I \in$ Ass $R$ iff $I Q \in$ Ass $Q$. This also implies that if $K$ is an ideal of $Q$, then $K \cap R \in$ Ass $Q$ iff $K \in$ Ass $R$ and, hence, that

$$
\text { (Ass prime } \operatorname{rad} Q) \cap R=\text { Ass } \operatorname{rad} R
$$

and

$$
\text { (Ass prime } \operatorname{rad} R) Q=\text { Ass } \operatorname{rad} Q \text {. }
$$

Moreover, $\left\{I_{i}\right\}_{i \in \Lambda}$ is a collection of independent ideals in $R$ iff $\left\{I_{i} Q\right\}_{i \in \Lambda}$ is the same in $Q$.

Therefore, $R$ has acc $\perp$ (resp., acc $\oplus$ ) iff $Q$ has the same property, and, by the above note, $Q$ is (semilocal) Kasch under either assumption: $Q$ Artinian or $R$ Goldie. Therefore, by Proposition 2.1, $Q$ has nil Jacobson radical iff $R$ has nil Ass $\operatorname{rad} R=\operatorname{Nil} \operatorname{rad} R$. Then Theorem 1.1 applies to conclude the proof.

2.3. Corollary. If $R$ is a Goldie ring with nil associated prime radical, then the polynomial ring $R[x]$ over $R$ is Goldie with nil associated prime radical.

Proof. By the theorem, $Q=Q_{c}(R)$ is Artinian and, hence,

$$
Q_{2}=Q_{c}(R[x])=Q_{c}(Q[x])
$$

is Noetherian and, in fact, Artinian by a theorem of Small [S1], but this also follows from Theorem 3.13 of [F2] which asserts for $Q$ semilocal Kasch that $J\left(Q_{2}\right)$ is nil when (and only when) $J(Q)$ is. Then, by the theorem, $Q_{2}$ is Artinian; hence, Ass $\operatorname{rad} R$ is nilpotent.

2.4. Corollary. Let $R$ be a Goldie Kerr ring. Then $\operatorname{rad} Q_{c}(R[x])$ is nil iff $Q_{c}(R[x])$ is Artinian and iff $Q_{c}(R)$ is Artinian.

Proof. The proof follows from Theorem 1.1, Theorem 1.2, and Corollary 2.3.

This corollary justifies a remark made in [F2] to the effect that while $\operatorname{rad} R[x]$ is nil, for any ring $R$, that $J_{2}=\operatorname{rad} Q_{2}$ is not necessarily nil.

2.5. Theorem. An algebraic Goldie algebra $R$ over a field $k$ is Artinian.

Proof. It is known in an algebraic algebra $R$ over a field $k$ that every nonzero divisor (= regular element) is a unit, that is, that $Q=Q_{c}(R)$. Moreover, by [J, p. 19, Theorem 1 and Corollary], $J(R)$ is a nil ideal, so $R$ is Artinian by Theorem 2.2.

Theorem 1.1 therefore implies that any non-Artinian Goldie algebra over a field is transcendental, and in fact, in any algebra over a field, the elements of $J(R)$ are either nilpotent or transendental (cf. [J, loc. cit.]).

2.6. Corollary. If $A$ is a Goldie ring and $Q_{c}(A)$ is an algebraic algebra over a field, then $A$ is a Kerr ring.

Proof. In general, a ring $A$ is Goldie iff $Q_{c}(A)$ is Goldie, so $Q_{c}(A)$ is Artinian by Theorem 2.5 , so $A$ is Kerr.

2.7. Corollary. If $A$ is a Goldie ring and $Q=Q_{c}(A)$ is an algebra over a field $k$ of cardinality exceeding the dimension of $Q$, then $Q$ is Artinian; hence, $A$ is Kerr.

Proof. Same proof as for Corollary 2.6 using a theorem of Amitsur [J, p. 20, Theorem 2] to deduce the $J(Q)$ is nil. 


\section{Commutative Rings With acc $\perp$}

What happens if you drop half of the two Goldie conditions and consider rings just with $\operatorname{acc} \perp$ ?

3.1. Theorem. If $R$ is an acc $\perp$ quotient ring with nil radical, then $R$ is semiprimary.

Proof. In the proof of Theorem 1.1, using acc $\perp$ we first deduce that $R$ is semiprimary, and we use acc $\oplus$ only to conclude that $R$ is finitely embedded.

3.2. Corollary. If $R$ is an acc $\perp$ ring with

Ass prime $\operatorname{rad} R=\operatorname{nil} \operatorname{rad} R$,

then $Q_{c}(R)$ is semiprimary.

Proof. The proof follows in the same way as the proof of the theorem.

As noted in [F2, Corollary 3.7], an acc $\perp$ ring $R$ has semilocal Kasch quotient ring $Q$. This is needed below.

3.3. Theorem (Added in proof). A reduced acc $\perp$ ring $R$ is Kerr, in fact $Q$ is semisimple Artinian.

Proof. Since $R$ is reduced, $R$ has zero singular ideal, hence $R$ has von Neumann regular $(=\mathrm{VNR})$ maximal quotient ring $Q_{m}=Q_{\max }(R)$ (see, e.g., [La, p. 106, Proposition 2]). However, $Q_{m} \supseteq Q$ canonically, and the fact noted above that $Q$ is Kasch implies that $Q=Q_{m}$ (cf. [La, p. 113, Exercise 5]). Since a semilocal VNR ring $Q$ is semisimple Artinian, we see that $R$ is Kerr.

3.4. Theorem (Added in proof). A valuation ring $R$ with acc $\perp$ has Noetherian quotient ring $Q$, and hence is Kerr.

Proof. By a theorem of Facchini (personal communication, anthologized in [FP, Corollary 6.11, p. 96]), any valuation ring $R$ has FP-injective quotient ring $Q$, and hence every finitely generated ideal of $Q$ is an annihilator ideal, so the acc $\perp$ in $R$, whence in $Q$, implies that $Q$ is Noetherian.

3.5. Remarks (Added in proof). 1 . If $R$ is a ring with just one nontrivial annihilator ideal, e.g., if $R$ is a local ring with $J^{2}=0$, then the same is true of any polynomial ring $R[X]$, in any number of variables; that is, $I[X]$ is the only nontrivial annihilator ideal. In fact, associated prime ideals of any ring $R$ extend to associated prime ideals of $R[X]$, and the correspondence is bijective [F2, Theorem 3.11].

2. If $R$ is a ring with zero-dimensional $Q$, that $Q$ is Artinian iff $R$ is Goldie follows from the results above and the fact that $J(Q)$ is nil.

\section{Notes}

1. A theorem of Camillo [C] characterizes Noetherian $R$ via Goldie rings: $R$ is Noetherian iff every factor ring is Goldie.

2. A theorem of Camillo and Guralnick [CG] shows that any acc $\perp$ ring $R$ that is an algebra over a nondenumerable field is a Kerr ring. They prove that this holds more generally if $R$ is a ring whose center contains a nondenumerable set $S$ with $s-t$ regular for every $s \neq t \in S$.

3. The explicit statement of Corollary 1.2 was suggested by the referee. 
4. Kerr [K2] constructed the first Goldie ring $R$ not embeddable in a Noetherian ring. Moreover, the ring $R$ is a Kerr algebra over an arbitrary field.

5. If $Q_{2}=Q_{c}(R[x])$ is self-injective, then $Q_{2}$ and $Q=Q_{c}(R)$ are $\mathrm{QF}$ by a theorem of Herbera and Pillay [HP].

6. See $[R]$ in connection with acc $\perp$ rings and Kerr and Camillo-Guralnick rings. Also, see [S2] in connection with embedding rings in Artinian rings.

7. F. Cedó has constructed a Kerr ring $R$ over which the power series ring $R\langle x\rangle$ does not have acc $\perp$ (e-mail communication).

\section{OPEN QUESTIONS}

1. Characterize Kerr rings.

2. If $R$ is a Kerr ring, is $R[x]$ ?

3. The answer to 2 is "yes" for the Camillo-Guralnick Kerr rings.

\section{REFERENCES}

[C] V. Camillo, Commutative rings whose quotients are Goldie, Glasgow Math. J. 16 (1975), 32-33.

[CG] V. Camillo and R. Guralnick, Polynomial rings are often Goldie, Proc. Amer. Math. Soc. 98 (1986), 567-568.

[F1] C. Faith, Finitely embedded commutative rings, Proc. Amer. Math. Soc. 112 (1991), 657-659; Addendum 118 (1993), 331.

[F2] _ Annihilators, associated prime ideals, and Kasch-McCoy quotient rings of commutative rings, Comm. Algebra 119 (1991), 1867-1892.

[F-P] C. Faith and P. Pillay, Classification of commutative FPF rings, Notas de Matematicas, vol. 4, Murcia: Universidad, Secretario de Publicaciones, 1990.

[GM] S. M. Ginn and P. B. Moss, Finitely embedded modules over Noetherian rings, Bull. Amer. Math. Soc. 49 (1975), 294-296.

[HP] D. Herbera and P. Pillay, Injective classical quotient rings of polynomial rings are quasiFrobenius, J. Pure Appl. Algebra 86 (1993), 51-63.

[HS] I. N. Herstein and L. Small, Nil rings satisfying certain chain conditions, Canad. J. Math. 16 (1964), 771-776.

[J] N. Jacobson, The structure of rings, Amer. Math. Soc., Providence, RI, 1956; revised ed. 1964.

[K1] J. W. Kerr, The polynomial ring over a Goldie ring need not be a Goldie ring, J. Algebra 134 (1990), 344-352.

[K2] __ An example of a Goldie ring whose matrix ring is not Goldie, J. Algebra 61 (1979), 590-592.

[La] J. Lambek, Lectures on rings and modules, Chelsea, New York, 1976.

[Le] J. Levitzki, On nil subrings, Israel Math. J. 7 (1963), 215-216.

[P] C. Perelló (ed.), Pere Menal Memorial volumes, Publ. Mat. 36 (2A \& 2B) (1992).

[R] M. Roitman, On Mori domains and commutative rings with $C C^{\perp}$. III, J. Pure Appl. Algebra 64 (1990), 315-328.

[S1] L. Small, Orders in Artinian rings, J. Algebra 4 (1966), 13-41.

[S2] The embedding problem for Noetherian rings, Bull. Amer. Math. Soc. 75 (1969), 147-148.

Department of Mathematics, Rutgers University, New Brunswick, New Jersey 08903 Permanent address: 199 Longview Drive, Princeton, New Jersey 08540 